\title{
Forced Arterial Suction Thrombectomy Using Distal Access Catheter in Acute Ischemic Stroke
}

\author{
Ho-Cheol Lee, MD', Dong-Hun Kang, MD', Yang-Ha Hwang, MD', \\ Yong-Sun Kim, MD³, Yong-Won Kim, MD ${ }^{1,3}$
}

Historical innovations in mechanical thrombectomy devices and strategies for ischemic stroke have resulted in improved angiographic outcomes and better clinical outcomes. Various devices have been used, but the two most common approaches are aspiration thrombectomy and stent-retrieval thrombectomy. Aspiration thrombectomy has advanced from the traditional Penumbra system to forced arterial suction thrombectomy and a direct aspiration first-pass technique. Newer generation aspiration catheters with flexible distal tips and a larger bore have demonstrated faster and better recanalization relative to older devices. Recently, several species of distal access catheters have similar structural characteristics to the Penumbra reperfusion catheter. Therefore, we used the distal access catheter for forced arterial suction thrombectomy in three patients with acute ischemic stroke. In each case, we achieved fast and complete recanalization without significant complications. Forced arterial suction thrombectomy using a distal access catheter might provide another option for mechanical thrombectomy in patients with acute ischemic stroke.

Key Words : Acute ischemic stroke; Mechanical thrombectomy;

Forced arterial suction thrombectomy; Aspiration thrombectomy; Distal access catheter

Since the release of the Merci retriever, mechanical thrombectomy devices have rapidly developed [1]. Along with advancement in mechanical thrombectomy

Departments of ${ }^{1}$ Neurology, ${ }^{2}$ Neurosurgery, and ${ }^{3}$ Radiology, Kyungpook National University Hospital, School of Medicine, Kyungpook National University, Daegu, Korea

Received December 28, 2016;

accepted after revision January 22, 2017.

Correspondence to: Yong-Won Kim, MD, Department of Neurology, Kyungpook National University Hospital, School of Medicine, Kyungpook National University, 130, Dongduk-ro, Junggu, Daegu 41944, Korea.

Tel. 82.53.420.5765 Fax. 82.53.422.4265

E-mail: yw.kim23@gmail.com

This is an Open Access article distributed under the terms of the Creative Commons Attribution Non-Commercial License (http://creativecommons.org/licenses/by-nc/3.0) which permits unrestricted non-commercial use, distribution, and reproduction in any medium, provided the original work is properly cited. devices and strategies, recent trials have demonstrated a greater efficacy and safety regarding the endovascular treatment of acute ischemic stroke (AIS) in the proximal anterior circulation occlusion [2-6]. Based on these results, recent guidelines have recommended endovascular treatment with a stent retriever for patients with an occlusion in the proximal intracranial artery [7].

Various devices have been used for mechanical thrombectomy, but the two most popular strategies are aspiration thrombectomy and stent-retrieval thrombectomy. For aspiration thrombectomy, the most recent generation of catheters, with their flexible distal tips and a larger bore, have demonstrated faster and better recanalization in comparison to older devices [8]. Recently, several distal access catheters (DAC) bear structurally similar characteristics to the Penumbra 


\section{Ho-Cheol Lee, et al.}

reperfusion catheter, and, further, have a diameter that is larger than the Penumbra reperfusion catheter. Based on this, we applied the DAC as an aspiration thrombectomy device in three patients with AIS. To our knowledge, there are no published reports of aspiration thrombectomy with a DAC in South Korea. Thus, we report three cases of forced arterial suction thrombectomy (FAST) with a DAC.

\section{CASE PRESENTATION}

Three patients were admitted to our hospital with neurological deficits within 3 hours from symptom onset. They all presented with a high National Institutes of Health Stroke Scale (NIHSS) score and brain computed tomography (CT) that showed no hemorrhagic density. Intravenous tissue plasminogen activator was administered, and CT angiography (CTA) and diffusion-weighted magnetic resonance imagining (DWI) were subsequently performed. After obtaining informed consent, mechanical thrombectomy was conducted under conscious sedation. Detailed information of each case is summarized in Table 1.

\section{Case 1}

A 47-year-old female patient was admitted to our hospital with left limb weakness. She had no prior medical history of hypertension, diabetes, or atrial fibrillation. The initial neurologic examination demonstrated a drowsy state, left hemiparesis, and dysarthria with a NIHSS score of 8 . Her vital signs were stable with a blood pressure of 170/90 $\mathrm{mmHg}$ and a serum glucose level of $71 \mathrm{mg} / \mathrm{dL}$. Non-enhanced brain CT revealed no hemorrhagic density and CTA showed an occlusion in the distal basilar artery (Fig. 1A). DWI revealed a subtle high signal in the right pons.

Endovascular mechanical thrombectomy was performed under conscious sedation. A 6-Fr guide catheter (Chaperon, MicroVention, Tustin, CA, USA) was introduced from the right femoral artery to the distal V2 segment in the left vertebral artery. A DAC (Navien 058; ev3/covidien, Irvine, CA, USA) coaxilally with a .021-inch microcatheter (Prowler select plus; Cordis, Miami, FL, USA) and a .014-inch microguidewire (Synchro-14; Stryker, Kalamazoo, MI, USA) was advanced to the proximal end of the occluded segment (Fig. 1G). After retraction of the microcatheter and microguidewire, direct aspiration with the DAC was performed using a $50-\mathrm{mL}$ syringe. The retrieved catheter showed a large thrombus wedged within it, and complete reperfusion was achieved (Fig. $1 \mathrm{~J})$. One-day follow-up with brain CT revealed no hemorrhagic complications.

Table 1. Detailed Information of Each Case

\begin{tabular}{|c|c|c|c|}
\hline Case No. & Case 1 & Case 2 & Case 3 \\
\hline Sex/Age & $\mathrm{F} / 47$ & $\mathrm{M} / 71$ & $\mathrm{~F} / 71$ \\
\hline Initial NIHSS & 7 & 19 & 30 \\
\hline Time from onset to door & $40 \mathrm{~min}$ & $83 \mathrm{~min}$ & $41 \mathrm{~min}$ \\
\hline Occlusion location & Distal BA & Left MCA M1 & Distal BA \\
\hline Used DACs & Navien 058 & Catalyst 6 & Sofia \\
\hline \multicolumn{4}{|l|}{ Angiographic time interval } \\
\hline Femoral puncture to reperfusion & $11 \min$ & $13 \min$ & $19 \min$ \\
\hline Guide catheter to target access & $2 \min 20 \mathrm{sec}$ & $1 \mathrm{~min} 25 \mathrm{sec}$ & $40 \mathrm{sec}$ \\
\hline Target access to reperfusion & $1 \mathrm{~min} 19 \mathrm{sec}$ & $2 \min 24 \mathrm{sec}$ & $2 \min 14 \mathrm{sec}$ \\
\hline Number of aspiration thrombectomy & 1 & 1 & 1 \\
\hline Final reperfusion grade (TICI) & 3 & 3 & 3 \\
\hline Hemorrhagic complication & None & None & None \\
\hline 7 day NIHSS & 0 & 4 & 1 \\
\hline
\end{tabular}

BA, Basilar artery; DAC, Distal access catheter; Min, Minute; NIHSS, National Institute of Health Stroke Scale; Sec, Second; TICI, Thrombolysis In Cerebral Infarction. 


\section{Case 2}

A 71-year-old male patient with a medical history of hypertension and hyperlipidemia was admitted to our hospital with right limb weakness. On arrival, his blood pressure was $115 / 65 \mathrm{mmHg}$, his blood sugar level was $113 \mathrm{mg} / \mathrm{dL}$, and electrocardiography showed a sinus rhythm. Initial neurologic examination revealed right facial palsy, global aphasia, and right hemiparesis with a NIHSS score of 19 . Non-enhanced brain CT revealed no hemorrhagic density, and CTA showed an occlusion of the M1 segment of the left MCA (Fig. 1B).

A 9-Fr balloon guide catheter (Optimo; Tokai Medical, Aichi, Japan) was advanced from the right femoral artery to the left cervical ICA. Steam shaping of the tip of the DAC (Catalyst 6; Stryker, Kalamazoo, MI, USA) was performed to facilitate passage through the ophthalmic segment. Under roadmap guidance, the
DAC with a .021-inch microcatheter and a .014-inch microguidewire (Traxcess 14 EX; MicroVention, Tustin, CA, USA) was advanced to the proximal M1 segment of the left MCA (Fig. 1H), and the microcatheter and microguidewire were removed. The DAC was wedged to the thrombus with direct manual aspiration using a 50-mL syringe. The BGC was inflated after confirming arrest of blood aspiration into the syringe, and the DAC was gently retrieved maintaining manual aspiration. During retrieval of the DAC, blood was aspirated into the syringe and the aspirate contained a large thrombus. Follow-up angiography revealed complete reperfusion (Fig. 1K). There was no hemorrhagic complication on the 1-day follow-up brain CT.

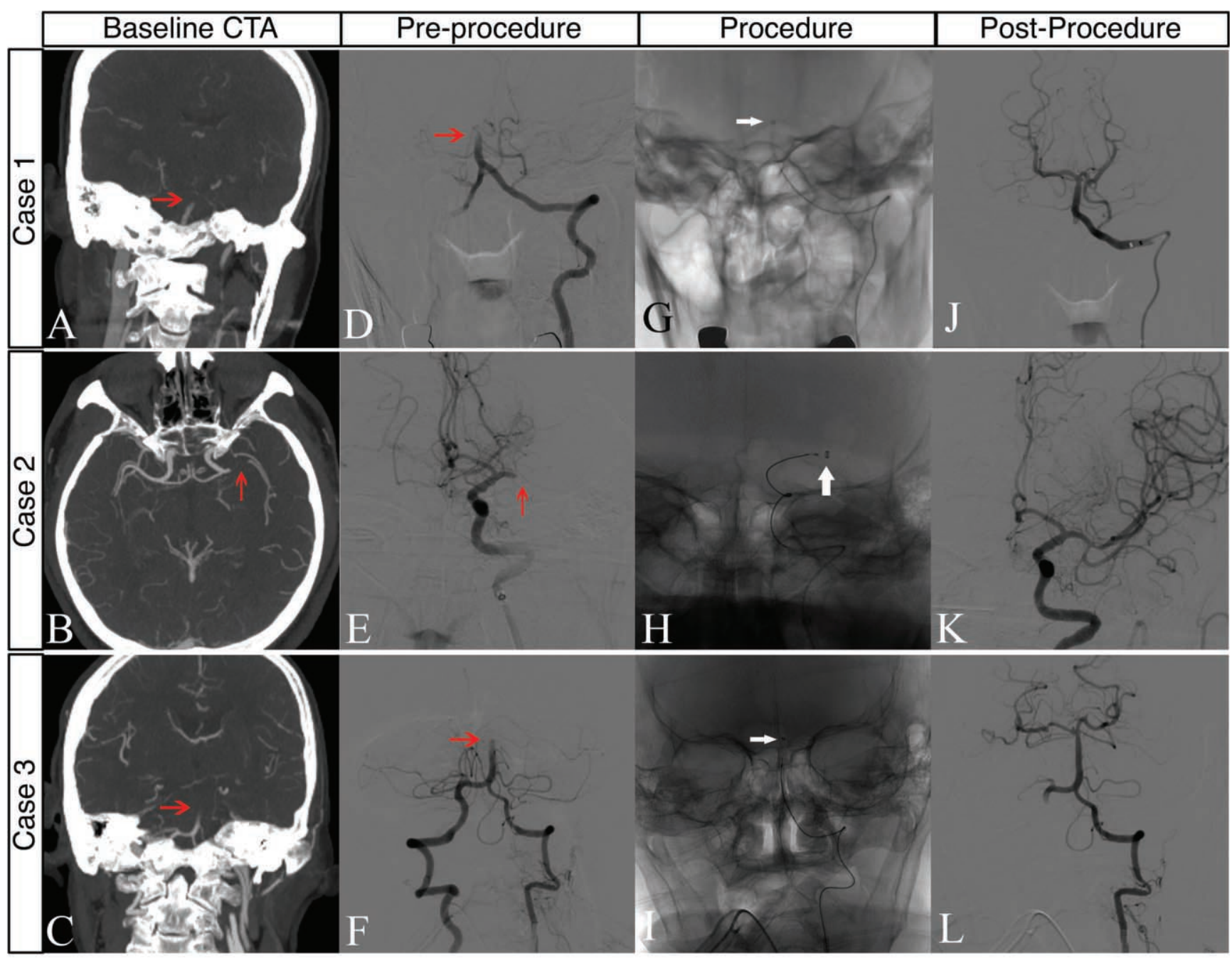

Fig. 1. Summary of baseline and angiographic images. Baseline CTA (A-C) and pre-procedural cerebral angiography (D-F) of 3 cases show an occlusion on intracranial artery (red arrow). The procedural cerebral angiography (G-I) demonstrates target access of distal access catheter (white arrow). The post-procedural angiography $(\mathbf{J}-\mathbf{L})$ shows successful reperfusion after aspiration thrombectomy using distal access catheter. 


\section{Ho-Cheol Lee, et al.}

\section{Case 3}

A 71-year-old female patient with no medical history of hypertension, diabetes mellitus, or arrhythmia was admitted to our hospital. The initial neurologic examination revealed a stuporous mental status and quadriparesis with a NIHSS score of 28. On admission, her blood pressure was $134 / 69 \mathrm{mmHg}$, her blood glucose level was $110 \mathrm{mg} / \mathrm{dL}$, and electrocardiography revealed sinus tachycardia. Non-enhanced brain CT was unremarkable, and CTA showed an occlusion of the distal basilar artery (Fig. 1C).

A 6-Fr guide catheter (Envoy; Cordis, Miami, FL, USA) was positioned from the right femoral artery to the distal V2 segment of the left vertebral artery. Under roadmap guidance, the 5-Fr DAC (Sofia; MicroVention, Tustin, CA, USA) with a .021-inch microcatheter and a .014-inch microguidewire was advanced to the proximal end of the occluded basilar artery (Fig. 1I), and the microcatheter and microguidewire were removed. And then, direct manual aspiration using a $50-\mathrm{mL}$ syringe through the DAC was conducted and a large thrombus was aspirated into the syringe. Follow-up angiography revealed complete reperfusion (Fig. 1L). On 1-day follow-up, brain CT showed no hemorrhagic density.

\section{DISCUSSION}

Theoretically, aspiration thrombectomy may be generally safe due to the rarity of vessel wall irritation or damage [9], and the use of a large diameter DAC might improve the wedging and extracting of a thrombus [10], which may in turn lead to better reperfusion results $[11,12]$. In this case series, we presented FAST using various DACs in patients with AIS. Successful reperfusion was achieved in all cases. There were no complications related to the endovascular procedure.

With the continuing evolution of mechanical thrombectomy devices, aspiration thrombectomy strategies have advanced from the traditional Penumbra aspiration system to Forced Arterial Suction Thrombectomy (FAST) and A Direct Aspiration FirstPass Technique (ADAPT) $[8,13,14]$. Generally, mechanical thrombectomy in patients with AIS is a time sensitive procedure, and successful recanalization within a short procedure time is important for increasing the likelihood of a good outcome. Direct aspiration strategies such as FAST and ADAPT may be advantageous with respect to fast, repetitive attempts during a procedure, because aspiration thrombectomy does not need stent re-sheathing and re-selection for an occluded artery. The critical advancement in aspiration thrombectomy devices was to increase the catheter diameter. Based on previous reports of mechanical thrombectomy using a Penumbra reperfusion catheter, new generation devices (5MAX ACE, 5MAX; Penumbra, Alameda, CA, USA) having a larger diameter have demonstrated a faster (36.6 vs $41.2 \mathrm{~min}$ ) and higher rate of TICI $2 \mathrm{~b} / 3$ reperfusion $(81.8 \%$ vs $95 \%)$ in comparison to old generation devices ( 041 , 032) $[8,14]$. Our aspiration thrombectomy strategy using DACs was also based on the idea that the aspiration force is proportional to the cross-sectional area of the catheter, and forceful aspiration with a largediameter catheter is advantageous with respect to wedging and extracting the thrombus [10]. We therefore attempted aspiration thrombectomy with 3 different kinds of large bore DACs, all of which had a larger inner diameter in comparison to the Penumbra $5 \mathrm{MAX}$ reperfusion catheter. Successful reperfusion was achieved with each kind of DAC.

Despite the advantage of aspiration thrombectomy using a large bore catheter, we needed to consider several factors. First, there might have been a difficulty in delivering the aspiration catheter to the target occlusion. Passage through the ophthalmic segment of the ICA might present a challenge with respect to accessing to the target. Manufacturers designed the distal part of the DAC to be soft and flexible to improve trackability. We used a coaxial technique with a .021inch microcatheter and steam-shaped the distal tip of the DAC. In case 2, a .060-inch DAC was delivered to the MCA without difficulty. A second, larger guide catheter had to be introduced for the use of a large bore catheter. In cases of posterior circulation occlusion, selection of a guide catheter might be considered for a diameter of the vertebral artery. Aspiration thrombectomy with a Penumbra reperfusion catheter (5MAX, 4MAX) cannot be introduced into a conventional guide catheter, such as 6 or 7-Fr Envoy or Chaperon, because of the larger outer diameter (.080 inch). Despite an inner diameter larger than the Penumbra reperfusion catheter, the outer diameter of the DACs used in our cases was less than or equal to .070 inches, and could be applied.

In conclusion, FAST using a large bore distal access catheter may be a viable treatment option for AIS. However, we cannot guarantee the safety and efficacy of this strategy due to our limited number of cases. But regarding technical aspects, FAST with a DAC is simple, fast, and straightforward. Further investigation 


\section{Forced Arterial Suction Thrombectomy Using Distal Access Catheter in Acute Ischemic Stroke}

\section{is necessary to confirm these findings.}

\section{References}

1. Spiotta AM, Chaudry MI, Hui FK, Turner RD, Kellogg RT, Turk AS. Evolution of thrombectomy approaches and devices for acute stroke: a technical review. J Neurointerv Surg 2015;7:2-7

2. Berkhemer OA, Fransen PS, Beumer D, van den Berg LA, Lingsma HF, Yoo AJ, et al. A randomized trial of intraarterial treatment for acute ischemic stroke. N Engl J Med 2015;372:11-20

3. Goyal M, Demchuk AM, Menon BK, Eesa M, Rempel JL, Thornton J, et al. Randomized assessment of rapid endovascular treatment of ischemic stroke. N Engl J Med 2015;372:1019-1030

4. Campbell BC, Mitchell PJ, Kleinig TJ, Dewey HM, Churilov L, Yassi N, et al. Endovascular therapy for ischemic stroke with perfusion-imaging selection. N Engl J Med 2015;372:1009-1018

5. Jovin TG, Chamorro A, Cobo E, de Miquel MA, Molina CA, Rovira A, et al. Thrombectomy within 8 hours after symptom onset in ischemic stroke. N Engl J Med 2015;372:2296-2306

6. Saver JL, Goyal M, Bonafe A, Diener HC, Levy EI, Pereira VM, et al. Stent-retriever thrombectomy after intravenous t-PA vs. t-PA alone in stroke. N Engl J Med 2015;372:2285-2295

7. Powers WJ, Derdeyn CP, Biller J, Coffey CS, Hoh BL, Jauch EC, et al. 2015 American Heart Association/American Stroke Association Focused Update of the 2013 Guidelines for the Early Management of Patients With Acute Ischemic Stroke Regarding Endovascular Treatment: A Guideline for Healthcare Professionals
From the American Heart Association/American Stroke Association. Stroke 2015;46:3020-3035

8. Turk AS, Frei D, Fiorella D, Mocco J, Baxter B, Siddiqui A, et al. ADAPT FAST study: a direct aspiration first pass technique for acute stroke thrombectomy. J Neurointerv Surg 2014;6:260-264

9. Teng D, Pannell JS, Rennert RC, Li J, Li YS, Wong VW, et al. Endothelial trauma from mechanical thrombectomy in acute stroke. Stroke 2015;46:1099-1106

10. Hu YC, Stiefel MF. Force and aspiration analysis of the ADAPT technique in acute ischemic stroke treatment. J Neurointerv Surg 2016;8:244-246

11. Son S, Choi DS, Oh MK, Hong J, Kim SK, Kang H, et al. Comparison of Solitaire thrombectomy and Penumbra suction thrombectomy in patients with acute ischemic stroke caused by basilar artery occlusion. J Neurointerv Surg 2016;8:13-18

12. Kabbasch C, Möhlenbruch M, Stampfl S, Mpotsaris A, Behme D, Liebig T. First-line lesional aspiration in acute stroke thrombectomy using a novel intermediate catheter: initial experiences with the SOFIA. Interv Neuroradiol 2016;22:333-339

13. Penumbra Pivotal Stroke Trial Investigators. The penumbra pivotal stroke trial: safety and effectiveness of a new generation of mechanical devices for clot removal in intracranial large vessel occlusive disease. Stroke 2009;40:2761-2768

14. Kang DH, Hwang YH, Kim YS, Park J, Kwon O, Jung C. Direct thrombus retrieval using the reperfusion catheter of the penumbra system: forced-suction thrombectomy in acute ischemic stroke. AJNR Am J Neuroradiol 2011;32:283-287 